\title{
Systemic therapy for advanced gastroesophageal cancers: progress and pitfalls
}

\author{
Raisa J. Epistola ${ }^{1}$, Joseph Chao $^{2}$ \\ ${ }^{1}$ Department of Internal Medicine, Harbor UCLA Medical Center, Torrance, CA, USA; ${ }^{2}$ Department of Medical Oncology \& Therapeutics \\ Research, City of Hope Comprehensive Cancer Center, Duarte, CA, USA \\ Contributions: (I) Conception and design: All authors; (II) Administrative support: None; (III) Provision of study materials or patients: None X; (IV) \\ Collection and assembly of data: None; (V) Data analysis and interpretation: None; (VI) Manuscript writing: All authors; (VII) Final approval of \\ manuscript: All authors. \\ Correspondence to: Joseph Chao, MD. Department of Medical Oncology \& Therapeutics Research, City of Hope Comprehensive Cancer Center, \\ 1500 East Duarte Road, Duarte, CA 91010, USA. Email: jchao@coh.org.
}

\begin{abstract}
Advanced gastroesophageal cancer in which surgical resection is no longer appropriate is an aggressive malignancy with poor prognosis. This review provides an overview of the key trials that have led to the current standard of care, both highlighting progress with systemic cytotoxic and biological therapies, but also calling attention to pitfalls to assist practitioners in optimizing currently available treatments for their patients. This review surveys recent and ongoing trials and biomarker studies regarding the use of anti-HER2 agents, with increased recognition of molecular intratumoral heterogeneity confounding such targeted therapy strategies. We conclude with an overview of recent major trials incorporating immune checkpoint inhibitors among patients with metastatic and locally advanced gastroesophageal cancer and providing a framework for the discriminate application of these new therapies.
\end{abstract}

Keywords: Gastric cancer; gastroesophageal cancer; triplet therapy; HER2; PD-1 inhibitor

Received: 05 December 2019; Accepted: 15 January 2020; Published: 05 October 2020.

doi: $10.21037 /$ tgh.2020.01.10

View this article at: http://dx.doi.org/10.21037/tgh.2020.01.10

\section{Introduction}

Gastric cancer is the fifth most frequently diagnosed cancer and third leading cause of cancer-related death globally $(1,2)$. While incidence rates are comparatively low in North America, there are projected to be approximately 27,000 new cases and 11,000 gastric cancer related deaths in 2019 in the United States alone (1,3). Esophageal cancer ranks seventh in incidence and sixth in overall mortality worldwide, and there are projected to be 17,650 new cases and 16,080 deaths related to esophageal cancer in 2019 in the United States $(3,4)$. Both esophageal and gastric cancers have particularly high prevalence in East Asia, with Japan, Korea and China carrying the highest burden of disease $(2,4)$. This group of cancers has an estimated overall annual cost of over 20.6 billion US dollars in Europe, Asia, North America and Australia combined (5). Despite a declining incidence over the last 50 years, prognosis is still poor with a $20 \% 5$ year survival rate and $74.5 \%$ fatality rate globally (2). Prognosis is worse in advanced disease, with a $5-10 \% 5$-year survival rate (5). Despite low survival rates with advanced disease, chemotherapy has been shown to improve health related quality of life (5). Given the challenges of treating this aggressive malignancy, this review aims to cover progress and pitfalls in the management of locally advanced and metastatic gastroesophageal cancer.

\section{Is there a role for triplet chemotherapy regimens in first-line gastroesophageal cancer?}

Historical studies seeking effective first-line systemic therapy options were predominantly empiric in nature with combining multiple cytotoxic agents. An example includes an early phase III trial comparing the efficacy 
of fluorouracil (5-FU), doxorubicin, methotrexate (FAMTX) therapy against epirubicin, cisplatin, and fluorouracil (ECF). This trial showed superiority in survival and response in 274 patients with inoperable adenocarcinoma or undifferentiated carcinoma of the esophagus, gastroesophageal junction (GEJ) or stomach in the ECF arm (6). Overall survival (OS) was improved with a median OS of 8.9 months in the ECF arm compared to 5.7 months in the FAMTX arm $(\mathrm{P}=0.0009)$, with significantly longer relapse free survival (RFS) in the ECF arm (7.4 vs. 3.4 months, $\mathrm{P}=0.00006)$. Overall objective response rates (ORR) were significantly improved in the ECF arm (45\% vs. $21 \%, \mathrm{P}=0.002)$, and thus this triplet entered into the regular treatment armamentarium. The REAL-2 trial was another early phase III non-inferiority trial which redefined triplet therapy among patients with inoperable or metastatic adenocarcinoma or squamous cell carcinoma (SCC) of the esophagus, GEJ, or stomach (7). A total of 1,002 patients were stratified to triple therapy with epirubicin, cisplatin, and fluorouracil (ECF); epirubicin, cisplatin, and capecitabine (ECX); epirubicin, oxaliplatin, and fluorouracil (EOF); or epirubicin, oxaliplatin, and capecitabine (EOX). The unadjusted hazard ratios (HR) for death in the capecitabine versus fluorouracil analysis was 0.86 [ $95 \%$ confidence interval (CI), 0.80 to 0.99 ] and 0.92 (95\% CI, 0.80 to 1.10 ) in the oxaliplatin versus cisplatin analysis, establishing the interchangeability of oxaliplatin with cisplatin as well as capecitabine with fluorouracil with regards to OS. As all four arms contained epirubicin, this study did not address epirubicin's contribution to therapeutic activity.

Another historical study addressing epirubicin's activity was a phase III clinical trial comparing ECF with MCF (mitomycin, cisplatin, fluorouracil) therapy (8). A total of 574 patients with inoperable adenocarcinoma, SCC or undifferentiated carcinoma of the esophagus, GEJ, or stomach were randomized to ECF $(n=284)$ or MCF $(n=285)$ therapy. ORR for ECF was $42.4 \%$ versus $44.1 \%$ for MCF $(\mathrm{P}=0.692)$. OS (9.4 months with ECF, 9.7 months with MCF, $\mathrm{P}=0.315)$ and failure free survival $(7$ months in both groups, $\mathrm{P}=0.572$ ) were similar. Thus, this study did not establish superior efficacy for either regimen in this mixed patient population. A Cochrane meta-analysis assessing the efficacy and tolerability of chemotherapy regimens among 501 patients with advanced gastric adenocarcinoma from 3 randomized trials, with inclusion of the adenocarcinoma subset from the aforementioned ECF vs. MCF study, concluded that three drug regimens consisting of 5-FU/ cisplatin/anthracycline, such as ECF, were superior to doublet 5-FU/cisplatin regimens with regards to OS (HR 0.77; 95\% CI, 0.62-0.91) (9). Yet, as this conclusion was drawn from a meta-analysis through pooled analyses of non-prespecified patient populations, there have been warranted critiques about broad application of this data establishing superior outcomes with prescribing triplet regimens containing an anthracycline, platinum, and fluoropyrimidine (10).

More recent data in the non-metastatic setting have provided some clarity of this potential pitfall of whether anthracycline-containing triplets should be prescribed routinely. OE05 was a phase III trial comparing cisplatin and fluorouracil (CF) neoadjuvant therapy with an escalated regimen of ECX among patients with surgically resectable esophageal and GEJ adenocarcinoma (11). A total of 451 patients were randomized to the CF group and 446 patients to the ECX group. The study demonstrated no significant differences in OS with an average median survival time of 23.4 months in the CF group versus 26.1 months in the ECX group $(\mathrm{P}=0.19)$. Importantly, a greater percentage of patients in the ECX group reported serious adverse events compared to the CF group $(\mathrm{P}=0.003)$. Providing supportive evidence of this conclusion in the metastatic setting was the CALGB 80403 randomized phase II study comparing efficacy and safety of ECF versus irinotecan and cisplatin (IC) versus oxaliplatin, fluorouracil, leucovorin (FOLFOX) in the first-line setting (12). Patients had treatment-naïve metastatic adenocarcinoma or SCC of the esophagus and GEJ with all 3 groups receiving the EGFR inhibitor cetuximab (C), as this trial was signalfinding at its conception to assist determination of which arm to carry forward in larger randomized studies. They concluded all three arms to be efficacious, determined by proportion of patients achieving complete (CR) or partial response $(\mathrm{PR})$ to therapy $(\mathrm{P}<0.001)$. Regarding toxicity, FOLFOX-C appeared to provide the most advantage with fewer treatment modifications compared with IC-C and ECF-C (73\%, 85\%, and 91\% respectively, $\mathrm{P}=0.013)$. Among the adenocarcinoma patients, which comprised $90 \%$ of the study population, median OS for ECF-C, IC-C, and FOLFOX-C was 11.6, 8.6, and 11.8 months. Median progression free survival (PFS) was 7.1, 4.9, and 6.8 months for ECF-C, IC-C, and FOLFOX-C groups. Fewer patients discontinued FOLFOX-C due to adverse events or treatment related death compared to IC-C and ECF-C, though this was not statistically significant $(11 \%, 26 \%, 19 \%$ respectively, 
Table 1 Summary of survival and toxicity outcomes in major taxane-containing triplet therapy trials

\begin{tabular}{llclll}
\hline Study & Study arms & Study N & ORR & Toxicity & Overall survival \\
\hline V325 & DCF vs. CF & 445 & $37 \%$ vs. $25 \%$ & G3-4 69\% vs. $59 \%$ & 9.2 vs. $8.6 \mathrm{mo}(\mathrm{P}=0.02)$ \\
Wang et al. & mDCF vs. CF & 243 & $48.7 \%$ vs. $33.9 \%$ & G53.4\% vs. $0.9 \%$ & 10.2 vs. $8.5 \mathrm{mo}(\mathrm{P}=0.03)$ \\
FLOT65+ & FLOT vs. FLO & 143 & $48.6 \%$ vs. $28.2 \%$ & G3-4 81.9\% vs. $38.6 \%$ & 17.3 vs. $14.5 \mathrm{mo}(\mathrm{P}=0.39)$ \\
Shah et al. & mDCF vs. DCF & 85 & $49 \%$ vs. $33 \%$ & G3-4 76\% vs. $90 \%$ & 18.8 vs. $12.6 \mathrm{mo}(\mathrm{P}=0.007)$ \\
\hline
\end{tabular}

Acronyms: CF (cisplatin, fluoruracil), DCF (docetaxel, cisplatin, fluoruracil), mDCF (modified docetaxel cisplatin, fluoruracil), FLOT (fluoruracil, leucovorin, oxaliplatin, doxetaxel), FLO (fluoruracil, leucovorin, oxaliplatin). ORR, objective response rate.

$\mathrm{P}=0.17)$. Hematologic toxicity (i.e., cytopenias) was similar across all 3 arms. While this trial was not powered to definitively define superiority, ORR, PFS and OS were not meaningfully different between the FOLFOX and ECF plus cetuximab regimens. In light of the OE05 trial results showing no benefit for OS and higher toxicity in the ECX triplet therapy arm, the NCCN guidelines have recommended two drug combinations such as fluorouracil with oxaliplatin or cisplatin if neoadjuvant chemotherapy is being considered for adenocarcinomas of the thoracic esophagus or GEJ (13). Indeed, expert opinions have recommended against epirubicin based treatment for all patients with advanced gastroesophageal adenocarcinoma, as epirubicin combinations have not shown clear benefits over two drug combinations, such as cisplatin and 5-FU $(13,14)$.

Another major effort to improve first-line triplet combinations was to incorporate taxanes with platinum and fluoropyrimidine chemotherapy agents. V325 was one of the initial phase III studies aimed to establish superiority of docetaxel, cisplatin, and fluorouracil (DCF) therapy over CF therapy with endpoints of time to progression (TTP) and OS (15). In this trial, 445 patients with metastatic or locally recurrent gastric and GEJ adenocarcinoma were randomized to DCF or CF therapy, with the trial meeting its primary endpoint of improving TTP in the experimental DCF arm. Specifically, the median TTP was 5.6 months for DCF compared to 3.7 months for CF (logrank $\mathrm{P}<0.001)$. There was a modest improvement in the secondary endpoint of OS: median OS was 9.2 months for DCF compared to 8.6 months for $\mathrm{CF}$ ( $\log$-rank $\mathrm{P}=0.02$; risk reduction $23 \%$ ). ORR was significantly higher in the DCF versus CF group as well (respectively $37 \%$ versus $25 \%$, $\mathrm{P}=0.01)$. Notably, increased efficacy appeared to come at a cost of higher toxicity with more grade 3 to 4 treatment related adverse events in the DCF (69\%) versus CF (59\%) treatment groups. Subsequent studies with taxane triplets have consistently shown improved ORR with addition of docetaxel, though there are variable outcomes with regards to improvement in OS, as summarized in Table 1 (16-18). The addition of a taxane to platinum and fluoropyrimidine doublets appears to be associated with a uniform increase in toxicity across multiple studies (15-17). Attempts to ameliorate side-effects by employing lowered doses and alternative schedules such as the modified DCF regimen have exhibited promising improved efficacy with less toxicities as standard DCF (18). A subsequent randomized phase III in China investigating a differing modified DCF protocol also exhibited promising improved OS versus a CF doublet, though 4 grade 5 events within 30 days of last treatment in the triplet arm versus 1 event in the doublet raises concerns regarding toxicity.

The more recent JCOG 1013 Trial was a phase III study comparing doublet therapy of S-1 (oral fluoropyrimidine derivative) and cisplatin (CS) versus triplet therapy of S-1, cisplatin and docetaxel (DCS). The study was conducted in Japanese centers, and was large and well powered, enrolling 741 patients with unresectable or recurrent gastric adenocarcinoma (19). The authors observed no significant differences between the DCS and CS group, even when separated by Lauren histology for the study's primary endpoint of OS. Median OS for all patients was 14.2 months in the DCS group and 15.3 months in the CS group $(\mathrm{P}=0.47)$. Separated by histologic type, OS was 13.3 months in the DCS group versus 14.2 months in the CS group among those with diffuse type histology $(\mathrm{P}=0.83)$ and 17.5 months in both the DCS and CS groups with intestinal type histology $(\mathrm{P}=0.65)$. Prognosis was poorer among Lauren diffuse subtype patients, in line with the medical literature, yet escalation to a taxane triplet did not improve outcomes. Furthermore, PFS was not significantly different between the DCS and CS groups (7.4 vs. 6.5 months, respectively, $\mathrm{P}=0.92)$; nor was ORR significantly different between DCS and CS $(59.3 \%$ vs. 56\% respectively, 
$\mathrm{P}=0.50$ ). This trial reported higher rates of grade 3 and 4 treatment related events in the DCS group. Capturing of patients prescribed post-trial second line chemotherapy was $79 \%$ in the CF group and $72 \%$ in the DCS group, which accounted for the majority of the patients and appeared balanced between both arms. Understandably, there was higher taxane second line therapy in the CS versus DCS arm (61\% vs. 29\%), but interestingly more patients in the CS versus DCS arm received ramucirumab (16\% vs. $6 \%$ ).

These multiple trials demonstrating increased adverse events of both epirubicin and docetaxel containing triplet therapy with varying efficacy outcomes suggest that it is not advantageous to prescribe all our active cytotoxic agents in first line therapy. The more recent JCOG 1013 trial further suggests any survival advantage would be annulled when the majority of patients are able to reach second-line therapy. Thus, what previously was considered progress may currently be considered a pitfall with second-line therapy options in gastroesophageal cancer being supported by multiple randomized trials and changing the treatment landscape in the past decade. Moving forward the rationale of two-drug versus three-drug therapies in the firstline setting appears two-fold, with equivalent survival by using our active cytotoxic agents sequentially, and further minimizing toxicities in first-line therapy to better afford our patients reaching second-line options.

\section{Should trastuzumab be used beyond progression on $1^{\text {st }}$ line trastuzumab containing therapy?}

The HER2 tyrosine kinase receptor became the first validated predictive biomarker for advanced gastroesophageal cancer based on the phase III ToGA trial investigating chemotherapy versus combination chemotherapy and trastuzumab among patients with locally advanced, recurrent or metastatic gastric and GEJ adenocarcinoma (20). These patients all had tumors that exhibited overexpression of the HER2 receptor tyrosine kinase by immunohistochemistry (IHC) or gene amplification detected by fluorescence insitu hybridization (FISH). A total of 594 patients were randomized to chemotherapy alone (doublet therapy of either capecitabine and cisplatin or flurouracil and cisplatin) or chemotherapy with the HER2 targeting monoclonal antibody trastuzumab. The study met its primary endpoint of improved OS in the combination therapy group. Median OS was 13.8 months in the trastuzumab group versus 11.1 months in the chemotherapy alone group $(\mathrm{P}=0.0026)$.
PFS was 6.7 vs. 5.5 months in the trastuzumab versus chemotherapy only treatment groups $(\mathrm{P}=0.0002)$. Posthoc analysis demonstrated greater clinical benefit for the subset of patients whose tumors exhibited a greater degree of overexpression of the HER2 receptor tyrosine kinase. Specifically, the margin of improved OS was greater with the addition of trastuzumab in patients with HER2 IHC scored as $2+$ with positive FISH or IHC scored as 3+ (16.0 versus 11.8 months, HR 0.65, 95\% CI, 0.51-0.83), contrasted with patients with tumor HER2 IHC scored as 0 or $1+$ despite being FISH positive (10.0 versus 8.7 months, HR 1.07, 95\% CI, 0.70-1.62).

Subsequent follow-up phase III studies investigated the efficacy of other HER2 targeted agents known to be effective in breast cancer, such as lapatinib, trastuzumab emtansine (T-DM1), and pertuzumab, among patients with advanced gastric and GEJ cancer. This included the TyTAN study, which compared second-line lapatinib and paclitaxel to paclitaxel monotherapy among patients with HER2 positive gastric cancer (21). There were no significant differences in primary endpoint of OS (11.0 months in lapatinib/paclitaxel versus 8.9 months in paclitaxel only, $\mathrm{P}=0.1044)$. There was no significant difference in PFS (5.4 months for lapatinib/paclitaxel arm versus 4.4 months for paclitaxel only; $\mathrm{P}=0.2441$ ), though there was higher ORR in the lapatinib group (27\% versus $9 \%$; OR $3.85 ; \mathrm{P}<0.001)$. Similarly, the LoGiC trial, which compared the efficacy of capecitabine/oxaliplatin (CapeOx) versus $\mathrm{CapeOx}$ with lapatinib among patients with HER2 positive advanced gastroesophageal adenocarcinoma found no significant difference in median OS (10.5 vs. 12.2 months; $\mathrm{P}=0.3492$ ) (22). There was no significant difference in the secondary outcome of PFS for the lapatinib plus CapeOx versus CapeOx only arms (6.0 vs. 5.4 months; $\mathrm{P}=.0381$ ), though there was a higher ORR in the lapatinib plus CapeOx arms ( $53 \%$ vs. $39 \%, \mathrm{P}=0.0031)$. Likewise, the JACOB trial was a phase III trial investigating the efficacy of adding pertuzumab to chemotherapy (cisplatin with capecitabine or $5-\mathrm{FU}$ ) and trastuzumab combination therapy among patients with metastatic gastric or GEJ cancer (23). OS was not significantly different between the treatment groups (17.5 months for pertuzamab/trastuzumab/chemotherapy arm versus 14.2 months for trastuzumab/chemotherapy arm; $\mathrm{P}=0.057)$. The pertuzumab group did have greater PFS (8.5 vs. 7.0 months; $\mathrm{P}=0.001)$ and ORR $(56.7 \%$ vs. $48.3 \% ; \mathrm{P}=0.026)$ when compared to the control group.

The GATSBY trial was a phase II/III study comparing the efficacy of second-line T-DM1 versus taxane 
monotherapy (either docetaxel or paclitaxel per physician preference) among patients with locally advanced or metastatic gastric and GEJ cancers (24). Median OS was 7.9 months with T-DM1 and 8.6 months with taxane treatment $(\mathrm{P}=0.86)$. There were no significant differences in PFS (2.7 months vs. 2.9 months in T-DM1 and taxane arms respectively (two-sided $\mathrm{P}=0.31$ ), nor were there significant differences in ORR (20.6\% for T-DM1 and $19.6 \%$ for taxane therapy; $\mathrm{P}=0.86)$. Notably, neither this study nor the aforementioned studies (TyTAN, LoGIC, JACOB) reached their primary endpoint of improved OS. A biomarker analysis of the GATSBY study investigated if there was any correlation with HER2 expression and the efficacy of T-DM1 (25). Importantly, confirmation of continued HER2 overexpression prior to starting second line therapy was not assessed in the prespecified biomarker analysis as fresh tumor biopsy was not mandatory for enrollment in the study. However, investigators did mandate central laboratory HER2 assessment be performed to minimize inter-laboratory variation. With a predominance of samples submitted for the biomarker analysis having come from archival formalin-fixed paraffin embedded (FFPE) specimens, the majority (90.4\%) of cases involved analysis of the primary tumor with the remaining analyzing metastatic or unknown site biopsies. Reflective of the intratumoral heterogeneity of HER2 overexpression in gastroesophageal cancer, $15.4 \%$ of patients demonstrated focal tumor HER2 staining, with homogenous HER2 staining observed in $56.7 \%$ of the analyzed cases. This analysis found that median OS was increased in subgroups with HER2 IHC $3+(9.5$ vs. 8.3 months for T-DM1 versus taxane; HR 0.99; 95\% CI, 0.68-1.43) vs. HER2 IHC 2+/in situ hybridizationpositive (5.2 vs. 9.2 months for T-DM1 versus taxane; HR 1.53 ; 95\% CI, 0.94-2.50) tumors. Trends towards increased median OS were observed in subgroups with greater than versus less than median HER2 mRNA expression, higher versus lower HER2 gene copy number, HER 2 gene ratio and $\mathrm{H}$ score, and homogenous or nonfocal HER2 IHC staining.

Trastuzumab has been shown to improve OS and PFS in previously treated patients with metastatic HER2 positive breast cancer. In addition to several phase III trials establishing that continuing anti-HER2 strategies (such as trastuzumab and T-DM1) beyond progression is effective, a randomized phase III study showed continuing even just trastuzumab beyond progression on initial trastuzumab while changing the chemotherapy backbone yielded improved median OS and PFS in breast cancer populations (26-28). Thus it is not surprising that many oncologists wanted to apply this approach to gastroesophageal cancer. A retrospective multicenter study assessed the efficacy of trastuzumab beyond progression among patients with HER2 positive advanced gastric carcinoma (29). It included 104 patients who had progressed on first line therapy of a platinum based agent and trastuzumab and compared the outcomes of patients who received second line therapy with trastuzumab $(n=39)$ with those who received second line therapy without trastuzumab $(n=65)$. Choice of second line chemotherapy was per discretion of the treating oncologist. This analysis showed improved OS (12.6 versus 6.1 months, $\mathrm{P}=0.001$ ) and $\mathrm{PFS}$ (4.4 versus 2.3 months, $\mathrm{P}=0.001$ ) among the group that continued trastuzumab therapy. While these results were compelling, as a retrospective analysis, this study is hypothesis generating only.

The phase II T-ACT trial compared paclitaxel alone with paclitaxel and trastuzumab therapy among patients $(\mathrm{n}=91)$ with HER-2 positive advanced gastric and GEJ cancer who had progressed after first line therapy with trastuzumab + fluoropyrimidine + platinum (30). This trial was the first to prospectively test whether continuing trastuzumab beyond progression on initial trastuzumab could be an effective strategy for gastric cancer as it is for breast cancer. Median PFS was 3.19 and 3.68 months in the paclitaxel and paclitaxel/trastuzumab arms, respectively $(\mathrm{P}=0.33)$ for their primary endpoint. The trial reported a median OS of 9.95 months in the paclitaxel arm and 10.2 months in the paclitaxel/trastuzumab arm $(\mathrm{P}=0.20)$. There was no significant difference in ORR and safety. Though only a small proportion of patients underwent re-biopsy of their tumors $(\mathrm{n}=16)$, it is compelling that this study observed high rates $(69 \%)$ of HER2 overexpression loss. Tumor biomarker evolution was further explored in a small, multicenter, prospective observational study re-evaluating biomarkers when acquired resistance to trastuzumab had emerged (31). All 33 patients enrolled in the study had been previously diagnosed with HER2 positive advanced or recurrent gastric adenocarcinoma that had progressed following firstline trastuzumab based therapy and were re-biopsied after progression to re-evaluate HER2 expression. This study found loss of HER2 overexpression in 20 patients $(60.6 \%)$ with refractory disease. These studies suggest that HER2 loss plays a major role in the development of acquired resistance to anti-HER2 therapy in gastric cancer.

Evidence that co-occurring genomic alterations influence anti-HER2 strategies was observed in a single arm, phase II trial $(n=32)$ that included cell-free DNA (cfDNA) analyses among patients with histologically confirmed HER2 positive 
metastatic and/or recurrent gastric adenocarcinoma (32) receiving CapeOx and lapatinib as first line therapy. They defined HER2 positivity as IHC $3+$ or IHC $2+$ with ERBB2 gene amplification by SISH (silver in situ hybridization). They met their primary endpoint for complete response (CR), reporting a CR rate of $21.8 \%$. The median PFS was 9.0 months and the median OS was 14.2 months. This trial included a biomarker analysis to better assess concomitant genomic alterations in HER2 positive patients. The most common alteration was CCNE1 amplification ( $40 \%$ of tumors). Though it did not meet the criteria for statistical significance, $66.7 \%$ of non-responders versus $22.2 \%$ of responders had CCNE1 amplification $(\mathrm{P}=0.08)$. Intuitively, ERBB2 which encodes HER2 was another biomarker associated with response to therapy, as patients with high level ERBB2 amplification were more likely to respond to therapy compared to patients with low level ERBB2 amplification $(\mathrm{P}=0.02)$. Notably, this study included 10 primary and metastatic tumor pairs that underwent tissue next generation sequencing (NGS). Of this group, 6 patients had concordant HER2 positive metastases, whereas 4 patients had discordant HER2 status of primary and metastatic tumors, emphasizing the heterogeneity of tumors within individual patients with gastroesophageal adenocarcinoma. Among 7 patients able to undergo postprogression biopsies, 3 (43\%) became categorized as HER2 negative on tissue testing. Paired cfDNA analyses provided an additional composite of temporal and spatial intrapatient heterogeneity of ERBB2 amplification and other genomic co-alterations correlating with treatment response and resistance, suggesting that cfDNA analyses may complement traditional tissue-based biomarker testing (33).

Researchers at Memorial Sloan Kettering performed a large case series with prospective biomarker analysis $(n=295)$ to identify biomarkers of drug sensitivity and resistance among patients with stage IV esophagogastric adenocarcinoma (34). They used both IHC/FISH and tissue NGS analysis for biomarker data. This study suggests the importance of using broad panel NGS in detecting genomic alterations in metastatic disease, given the heterogeneity of metastatic sites. They reported heterogeneity among ERBB2 amplification and RTK-RAS-PI3K/AKT pathway genes that were captured with NGS. For example, though there was a high concordance between IHC/FISH and NGS for ERBB2 copy number (positive predictive value of $90 \%$ and negative predictive value of $96.7 \%$ ), 4 patients (8\%) showed discordance, which was attributed to tumor heterogeneity and equivocal IHC/FISH positivity. Notably, these discordant cases exhibited significantly shorter PFS when compared to patients with ERBB2-amplified tumors by NGS (median PFS 5.8 versus 14.0 months; $\mathrm{P}=1 \mathrm{e}-6$ ). This study reported loss of HER2 positivity associating with trastuzumab resistance: in the 44 HER2 positive patients with post-trastuzumab samples, $7(16 \%)$ had lost or had significantly lower HER2 expression compared to pretreatment samples.

With better recognition of this pitfall that continuing anti-HER2 strategies beyond progression is not efficacious due to high rates of HER2 loss, what recourse do practitioners currently have for these patients in need of subsequent therapy? Current recommended secondline treatment irrespective of tumor HER2 status stems from the phase III RAINBOW trial comparing paclitaxel plus placebo to combination paclitaxel and ramucirumab among patients with advanced gastric or GEJ adenocarcinoma (35). Ramucirumab targets tumor angiogenesis through inhibition of the VEGFR2 pathway. A subgroup analysis of the RAINBOW trial analyzed the addition of ramucirumab to paclitaxel among patients who had received prior trastuzumab therapy (36). This analysis identified 39 patients who had inclusion of trastuzumab in their first-line therapy; 20 of which had been enrolled in the combination therapy arm and 19 of which had been enrolled in the placebo-control arm. There was a trend of longer OS in the combination therapy group, though this was not statistically significant (11.4 vs. 7.0 months; $\mathrm{P}=0.30$ ). The subgroup analysis did report higher median PFS (4.2 vs. 2.7 months; $\mathrm{P}=0.01$ ) and ORR $(45.0 \%$ vs. $10.5 \%, \mathrm{P}=0.07)$ in the combination therapy group compared to the placebo-paclitaxel control group. Given these observations and lack of further clinical trial data, paclitaxel with ramucirumab currently remains the standard of care for patients with HER2 positive advanced gastroesophageal cancer who have failed first line trastuzumab.

Despite lack of data showing improved OS in this population in clinical trials, enthusiasm remains in researching novel strategies to target the HER2 biomarker. Novel anti-HER2 strategies include re-engineered antiHER2 antibodies (i.e., margetuximab), anti-HER2 antibody drug conjugates (trastuzumab deruxtecan), newer generation HER2 tyrosine kinase inhibitors (tucatinib), combining with immune checkpoint inhibitors (pembrolizumab, avelumab), and cellular based therapies ( $\mathrm{T}$ cell and NK cells); a recent review cites at least 30 ongoing studies for these agents (37). Ongoing studies like clinicaltrials.gov NCT04014075 
examining trastuzumab deruxtecan therapy among patients with HER2 positive unresectable or metastatic gastric/ GEJ cancer after progression on trastuzumab now require re-biopsy of patients' tumors to confirm that HER2 overexpression is not lost before starting new HER2directed therapy (38).

\section{What is the current optimal use of PD-1 inhibitors in gastroesophageal cancer?}

Immune checkpoint inhibitors first garnered U.S. regulatory approval based on the KEYNOTE-059 phase II trial investigating the use of the PD-1 inhibitor pembrolizumab among patients with recurrent or metastatic gastric or GEJ adenocarcinoma who progressed after at least 2 prior chemotherapy regimens. This study included 259 patients and had a primary endpoint of ORR and safety (39). Among all patients, ORR was $11.6 \%$, with CR in $2.3 \%$. This study included a PD-L1 biomarker analysis and developed the PD-L1 IHC combined positive score (CPS) to determine PD-L1 positivity. CPS is calculated as the number of $\mathrm{PD}-\mathrm{L} 1$ positive cells divided by the total number of tumor cells, multiplied by 100 . Tumors were considered PD-L1 positive if the CPS was $\geq 1$. In subgroup analyses, ORR was $15.5 \%$ and $6.4 \%$ in patients with PD-L1-positive and PD-L1-negative tumors, respectively. Patients who received pembrolizumab as third-line treatment had ORR of $16.4 \%$ vs. $6.4 \%$ in patients who received pembrolizumab as fourth-line or later. Regarding safety outcomes, $17.8 \%$ experienced 1 or more grade 3 to 5 treatment-related adverse events, with 2 patients $(0.8 \%)$ discontinuing therapy due to treatment-related adverse events. The trial reported 2 deaths related to treatment. What ultimately garnered regulatory approval in the third-line and beyond setting is that response, if obtained, appeared durable, with 16 of 30 patients $(53.3 \%)$ exhibiting ongoing responses at trial publication and median duration of response ranging from 1.6 to 17.3 months.

The ATTRACTION-02 trial was the first randomized phase III trial of immune checkpoint inhibition in patients with advanced gastric or GEJ adenocarcinoma, which investigated the efficacy of nivolumab, a IgG4 monoclonal antibody inhibiting PD-1, in patients whose disease had progressed on 2 or more chemotherapy regimens (40). Patients were randomized to nivolumab $(\mathrm{n}=330)$ or placebo $(\mathrm{n}=163)$. Primary endpoint was median OS, which was 5.26 months in the nivolumab group and 4.14 months in the placebo group $(\mathrm{P}<0.0001)$. Median PFS was 1.61 months in the nivolumab group and 1.45 months in the placebo group $(\mathrm{P}<0.0001)$. The study included a post-hoc exploratory biomarker analysis of 142 patients who had tissue samples available. PD-L1 tumor expression was defined as staining of $1 \%$ or more of tumor cells by IHC, differing from the CPS criterion used in the KEYNOTE-059 study that included staining of immune cells. This exploratory analysis showed that median OS in patients with PD-L1-positive tumors was 5.22 months in the nivolumab group and 3.83 months in the placebo group (HR 0.51, 95\% CI, 0.21-1.25). Among patients with PD-L1-negative tumors, median OS was 6.05 months in the nivolumab group and 4.19 months in the placebo group (HR 0.72, 95\% CI, 0.49-1.05). Differential responses to a PD-1 inhibitor did not appear discernable between PD-L1 positive and PD-L1 negative groups when categorizing PD-L1 expression in tumor cells only. Nivolumab subsequently received regulatory approval in Japan as third-line therapy independent of biomarker testing.

The KEYNOTE-061 trial was the first randomized trial of an anti-PD-1/anti-PD-L1 agent as second line therapy versus an active comparator for advanced gastric cancer (41). This phase III trial compared the efficacy of pembrolizumab $(\mathrm{n}=196)$ to paclitaxel $(\mathrm{n}=199)$ among patients with CPS of 1 or higher advanced or metastatic gastric/GEJ adenocarcinoma that had progressed on first line therapy of platinum and fluoropyrimidine. All patients had histologically or cytologically confirmed adenocarcinoma and had to provide newly obtained (within 6 weeks of study participation) tumor samples for PD-L1 assessment, thus providing a more contemporaneous assessment of PD-L1 status. Primary endpoint was OS among patients with CPS $\geq 1$, which was reported as 9.1 months in the pembrolizumab arm and 8.3 months in the paclitaxel arm $(\mathrm{P}=0.0421)$. Among patients with $\mathrm{CPS}<1$, median OS was 4.8 months (95\% CI, 3.9-6.1 months) with pembrolizumab versus 8.2 months (95\% CI, 6.8-10.6 months) with paclitaxel, providing support that complete lack of tumor PD-L1 expression utilizing the CPS criterion can serve as an effective negative predictive biomarker for anti-PD-1 therapy. Post-hoc analysis of patients with CPS $\geq 10$ showed median OS of 10.4 months with pembrolizumab versus 8.0 months with paclitaxel (HR 0.64, 95\% CI, 0.41-1.02), though this observation remains hypothesis-generating. The RAINBOW trial, as previously discussed, was a phase III trial establishing greater efficacy of paclitaxel with ramucirumab over paclitaxel alone as second line therapy for locally advanced/metastatic gastric and GEJ cancer. Therefore, a critique can be raised if paclitaxel should be 
considered an adequate comparator as the control arm in the KEYNOTE-061 study, and premature prescribing of single agent PD-1 inhibitors in second-line should be an avoided pitfall.

KEYNOTE-062 was a phase III trial, only presented in abstract form at time of this review, investigating the role of pembrolizumab in first line therapy among patients with locally advanced or metastatic gastric/ GEJ adenocarcinoma with HER2 negative and PD-L1postive disease. PD-L1-positivity was defined as CPS $\geq 1$. Patients were randomized into three arms: pembrolizumab monotherapy; pembrolizumab and chemotherapy (cisplatin with 5 -FU or capecitabine); chemotherapy and placebo. Comparative analyses were made between pembrolizumab versus chemotherapy/placebo and pembrolizumab/ chemotherapy versus chemotherapy/placebo. The primary outcomes were OS and PFS and included subgroup analyses of CPS $\geq 10$ and CPS $\geq 1$. Pembrolizumab was noninferior to chemotherapy/placebo with reported OS of 10.6 months in the pembrolizumab arm versus 11.1 months in the chemotherapy arm in the CPS $\geq 1$ group (noninferiority margin 1.2). Among patients with CPS $\geq 10$, OS was improved with median OS of 17.4 months with pembrolizumab versus 10.8 months with chemotherapy. Pembrolizumab/chemotherapy was not superior to chemotherapy for OS in either the CPS $\geq 1$ (12.5 versus 11.1 months; $\mathrm{P}=0.046$ ) or $\mathrm{CPS} \geq 10$ arms ( 12.3 versus 10.8 months; $\mathrm{P}=0.158)$. Interestingly, $\mathrm{PFS}$ was inferior in the pembrolizumab versus chemotherapy analysis among CPS $\geq 1$ patients (2.0 versus 6.4 months; HR 1.66, 95\% CI, 1.37-2.01) querying the clinical applicability of this trial. However, a recent abstract presentation of the KEYNOTE-062 dataset reported improved OS and PFS among patients with microsatellite-instability high (MSI-H) tumors with CPS $\geq 1$ (42). Specifically, OS endpoints appeared greatly improved for both pembrolizumab versus chemotherapy (HR 0.29; 95\% CI, 0.11-0.81) and pembrolizumab/chemotherapy versus chemotherapy alone (HR 0.37; 95\% CI, 0.14-0.97) with median OS not having been reached for both pembrolizumab containing arms at last trial reporting. PFS was longer with pembrolizumab $v s$. chemotherapy (HR 0.72; 95\% CI, 0.31-1.68) and pembrolizumab/chemotherapy vs. chemotherapy (HR $0.45 ; 95 \%$ CI, 0.18-1.11). Both the aforementioned KEYNOTE-059 and KEYNOTE-061 trials had reported a high proportion of clinical benefit in patients with MSI-H tumors. While small subsets of each major randomized trial, the collection of data suggests that durable responses appear maintained across lines of therapy and progress for earlier use of pembrolizumab should be advocated in this molecular subset of gastric cancer.

In examining the use of immune checkpoint inhibitors in SCCs, the other major histologic subset of gastroesophageal cancers, KEYNOTE-181 was a phase III trial investigating the efficacy of pembrolizumab therapy in patients with advanced esophageal cancer who had progressed after first line chemotherapy (43). Patients with both esophageal SCC and adenocarcinoma histology as well as Siewert I GEJ adenocarcinomas were randomized to open label pembrolizumab and investigator's choice of paclitaxel, docetaxel, or irinotecan chemotherapy. Co-primary endpoints were OS in PD-L1 CPS $\geq 10$, OS in SCC patients, and OS in the entire ITT population. This trial was considered positive for the PD-L1 CPS $\geq 10$ endpoint $(\mathrm{N}=222$; median 9.3 versus 6.7 months; $\mathrm{P}=0.0074)$. While not statistically significant, they reported a clinically meaningful improvement in OS for the SCC population of patients $(\mathrm{N}=401 ; 8.2$ vs. 7.1 months; $\mathrm{P}=0.0095)$ with pembrolizumab versus chemotherapy. Difference in OS was not statistically significant for the ITT population, though it did trend favorably as well $(\mathrm{N}=628 ; 7.1 \mathrm{vs} .7 .1$ months; $\mathrm{P}=0.0560)$. Pembrolizumab subsequently gained U.S. regulatory approval specifically in SCC patients whose tumors demonstrate PD-L1 CPS expression $\geq 10$ bringing necessary progress for this histology.

Providing affirmation for single agent PD-1 inhibitors' effectiveness in esophageal SCC, ATTRACTION-3 was a phase III trial that randomized patients with unresectable or recurrent esophageal SCC patients to $2^{\text {nd }}$-line nivolumab, a PD-1 inhibitor, or investigator's choice of paclitaxel or docetaxel chemotherapy (44). The trial demonstrated OS improvement for nivolumab for the entire ITT study population with $>96 \%$ of patients of Asian descent. OS was significantly improved in the nivolumab group compared with the chemotherapy group (10.9 versus 8.4 months; $\mathrm{P}=0.019)$. However, PFS was not significantly different between the two arms with median PFS of 1.7 months in the nivolumab arm versus 3.4 months in the chemotherapy arm (HR 1.08, 95\% CI, 0.87-1.34). PD-L1 IHC as a biomarker was only examined in expression by tumor cells and did not differentiate benefit between nivolumab $v s$. chemotherapy. Patients with tumor PD-L1 expression $<1 \%$ had median OS of 10.9 months with nivolumab. Those with $\geq 1 \%$ PD-L1 expression had median OS of 10.9 months with nivolumab. Median OS in the chemotherapy arm was 9.3 and 8.1 months in the $<1 \%$ and $\geq 1$ groups, 
respectively. Whether a differential benefit to nivolumab would have been observed if a PD-L1 CPS criterion was applied remains an unanswered question. Together, the KEYNOTE-181 and ATTRACTION-3 studies suggest that histology (i.e., squamous $v$ s. adenocarcinoma) and site of origin (esophageal, GEJ, gastric body/distal stomach) play a role on response to single agent immune checkpoint inhibitors, such as nivolumab and pembrolizumab.

Given these apparent differences in responses to therapy, there has been ongoing research to better delineate these tumor types. Indeed, high throughput sequencing efforts have supported molecular differences of these tumors. The Cancer Genome Atlas Research Network conducted a comprehensive molecular analysis of 164 esophageal tumors, 359 gastric adenocarcinomas, and 36 adenocarcinomas of the GEJ in an effort to better demarcate gastric adenocarcinomas from esophageal adenocarcinomas and SCC (45). The analysis concluded that esophageal SCCs were more similar to SCCs of other organ sites than to esophageal adenocarcinomas. Furthermore esophageal carcinomas resembled the chromosomally unstable variant of gastric adenocarcinoma, though with some unique features, such as DNA hypermethylation. These data add to evidence that esophageal adenocarcinomas and SCCs are molecularly distinct entities, which must be taken into account in the development of future therapies and clinical trials.

\section{Conclusions}

Though advanced gastroesophageal cancer is an aggressive malignancy with poor prognosis, progress has been made in developing effective therapies to improve patient survival. In summary, doublet therapy is preferable over triplet therapy for first line treatment of advanced gastroesophageal cancer to minimize risks of drug toxicity and impact on quality of life in a predominantly non-curative setting. The continued use of trastuzumab and other anti-HER2 agents beyond first-line progression have garnered negative trial results to date for advanced gastroesophageal cancer, thus enforcing that outside of a trial setting this practice should be a pitfall. Similarly, results have been mixed regarding efficacy for PD-1 inhibitors in earlier lines of therapy with exception of positive progress for the MSI-H molecular subset of gastroesophageal adenocarcinomas. Molecular studies have revealed new challenges of treating gastroesophageal cancer, including temporal and intratumoral heterogeneity of genomic drivers, as seen in the HER2 literature, as well as the need to further differentiate gastric, esophageal, and
GEJ cancers on a molecular basis. Indeed, these molecular studies have highlighted the need to incorporate composite biomarker testing strategies to continue forward progress and minimize pitfalls of developing new therapies and clinical trials for gastroesophageal cancer.

\section{Acknowledgments}

The authors would like to acknowledge the important work of other investigators who were unable to be cited due to space constraints.

\section{Footnote}

Conflicts of Interest: J Chao has received consultant/advisory fees from Lilly Oncology, Astra-Zeneca, Merck, Boston Biomedical, and Foundation Medicine. J Chao has received research funding (institutional) from Merck, and serves on the speaker's bureau for Merck. The other author has no conflicts of interest to declare.

Ethical Statement: The authors are accountable for all aspects of the work in ensuring that questions related to the accuracy or integrity of any part of the work are appropriately investigated and resolved.

\section{References}

1. Bray F, Ferlay J, Soerjomataram I, et al. Global cancer statistics 2018: GLOBOCAN estimates of incidence and mortality worldwide for 36 cancers in 185 countries. CA Cancer J Clin 2018;68:394-424.

2. Balakrishnan M, George R, Sharma A, et al. Changing Trends in Stomach Cancer Throughout the World. Curr Gastroenterol Rep 2017;19:36.

3. Siegel RL, Miller KD, Jemal A. Cancer statistics, 2019 (US statistics). CA Cancer J Clin 2019;69:7-34.

4. Jemal A, Bray F, Center MM, et al. Global cancer statistics. CA Cancer J Clin 2011;61:69-90.

5. Casamayor M, Morlock R, Maeda H, et al. Targeted literature review of the global burden of gastric cancer. Ecancermedicalscience 2018;12:883.

6. Webb A, Cunningham D, Scarffe JH, et al. Randomized trial comparing epirubicin, cisplatin, and fluorouracil versus fluorouracil, doxorubicin, and methotrexate in advanced esophagogastric cancer. J Clin Oncol 1997;15:261-7.

7. Cunningham D, Starling N, Rao S, et al. Capecitabine and oxaliplatin for advanced esophagogastric cancer. $\mathrm{N}$ Engl J 
Med 2008;358:36-46.

8. Ross P, Nicolson M, Cunningham D, et al. Prospective randomized trial comparing mitomycin, cisplatin, and protracted venous-infusion fluorouracil (PVI 5-FU) with epirubicin, cisplatin, and PVI 5-FU in advanced esophagogastric cancer. J Clin Oncol 2002;20:1996-2004.

9. Wagner $\mathrm{AD}$, Grothe $\mathrm{W}$, Haerting J, et al. Chemotherapy in advanced gastric cancer: A systematic review and meta-analysis based on aggregate data. J Clin Oncol 2006;24:2903-9.

10. Ajani JA. Standard of care for gastric cancer based on meta-analysis? Treading on thin ice or it is very nice! J Clin Oncol 2006;24:5473-4.

11. Alderson D, Cunningham D, Nankivell M, et al. Neoadjuvant cisplatin and fluorouracil versus epirubicin, cisplatin, and capecitabine followed by resection in patients with oesophageal adenocarcinoma (UK MRC OE05): an open-label, randomised phase 3 trial. Lancet Oncol 2017;18:1249-60.

12. Enzinger PC, Burtness BA, Niedzwiecki D, et al. CALGB 80403 (Alliance)/E1206: A randomized phase II study of three chemotherapy regimens plus cetuximab in metastatic esophageal and gastroesophageal Junction Cancers. J Clin Oncol 2016;34:2736-42.

13. Ajani JA, D’Amico TA, Bentrem DJ, et al. Esophageal and Esophagogastric Junction Cancers, Version 2.2019, NCCN Clinical Practice Guidelines in Oncology. J Natl Compr Canc Netw 2019;17:855-83.

14. Elimova E, Janjigian YY, Mulcahy M, et al. It Is Time to Stop Using Epirubicin to Treat Any Patient With Gastroesophageal Adenocarcinoma. J Clin Oncol 2017;35:475-7.

15. Van Cutsem E, Moiseyenko VM, Tjulandin S, et al. Phase III study of docetaxel and cisplatin plus fluorouracil compared with cisplatin and fluorouracil as first-line therapy for advanced gastric cancer: A report of the V25 study group. J Clin Oncol 2006;24:4991-7.

16. Wang J, Xu R, Li J, et al. Randomized multicenter phase III study of a modified docetaxel and cisplatin plus fluorouracil regimen compared with cisplatin and fluorouracil as first-line therapy for advanced or locally recurrent gastric cancer. Gastric Cancer 2016;19:234-44.

17. Al-Batran SE, Pauligk C, Homann N, et al. The feasibility of triple-drug chemotherapy combination in older adult patients with oesophagogastric cancer: A randomised trial of the Arbeitsgemeinschaft Internistische Onkologie (FLOT65+). Eur J Cancer 2013;49:835-42.

18. Shah MA, Janjigian YY, Stoller R, et al. Randomized
Multicenter Phase II Study of Modified Docetaxel, Cisplatin, and Fluorouracil (DCF) Versus DCF Plus Growth Factor Support in Patients With Metastatic Gastric Adenocarcinoma: A Study of the US Gastric Cancer Consortium. J Clin Oncol 2015;33:3874-9.

19. Yamada Y, Boku N, Mizusawa J, et al. Docetaxel plus cisplatin and S-1 versus cisplatin and S-1 in patients with advanced gastric cancer (JCOG1013): an open-label, phase 3, randomised controlled trial. Lancet Gastroenterol Hepatol 2019;4:501-10.

20. Bang YJ, Van Cutsem E, Feyereislova A, et al. Trastuzumab in combination with chemotherapy versus chemotherapy alone for treatment of HER2-positive advanced gastric or gastro-oesophageal junction cancer (ToGA): A phase 3, open-label, randomised controlled trial. Lancet 2010;376:687-97.

21. Satoh T, Doi T, Ohtsu A, et al. Lapatinib plus paclitaxel versus paclitaxel alone in the second-line treatment of HER2-amplified advanced gastric cancer in Asian populations: TyTAN - A randomized, phase III study. J Clin Oncol 2014;32:2039-49.

22. Hecht JR, Bang YJ, Qin SK, et al. Lapatinib in combination with capecitabine plus oxaliplatin in human epidermal growth factor receptor 2-positive advanced or metastatic gastric, esophageal, or gastroesophageal adenocarcinoma: TRIO-013/LOGiC - A randomized phase III trial. J Clin Oncol 2016;34:443-51.

23. Tabernero J, Hoff PM, Shen L, et al. Pertuzumab plus trastuzumab and chemotherapy for HER2-positive metastatic gastric or gastro-oesophageal junction cancer (JACOB): final analysis of a double-blind, randomised, placebo-controlled phase 3 study. Lancet Oncol 2018;19:1372-84.

24. Thuss-Patience PC, Shah MA, Ohtsu A, et al. Trastuzumab emtansine versus taxane use for previously treated HER2positive locally advanced or metastatic gastric or gastrooesophageal junction adenocarcinoma (GATSBY): an international randomised, open-label, adaptive, phase $2 / 3$ study. Lancet Oncol 2017;18:640-53.

25. Shah MA, Kang YK, Thuss-Patience PC, et al. Biomarker analysis of the GATSBY study of trastuzumab emtansine versus a taxane in previously treated HER2-positive advanced gastric/gastroesophageal junction cancer. Gastric Cancer 2019;22:803-16.

26. Diéras V, Miles D, Verma S, et al. Trastuzumab emtansine versus capecitabine plus lapatinib in patients with previously treated HER2-positive advanced breast cancer (EMILIA): a descriptive analysis of final overall survival results from a randomised, open-label, phase 3 trial. 
Lancet Oncol 2017;18:732-42.

27. Krop IE, Kim SB, Martin AG, et al. Trastuzumab emtansine versus treatment of physician's choice in patients with previously treated HER2-positive metastatic breast cancer (TH3RESA): final overall survival results from a randomised open-label phase 3 trial. Lancet Oncol 2017;18:743-54.

28. von Minckwitz G, du Bois A, Schmidt M, et al.

Trastuzumab beyond progression in human epidermal growth factor receptor 2-positive advanced breast cancer: a german breast group 26/breast international group 03-05 study. J Clin Oncol 2009;27:1999-2006.

29. Palle J, Tougeron D, Pozet A, et al. Trastuzumab beyond progression in patients with HER2-positive advanced gastric adenocarcinoma: A multicenter AGEO study. Oncotarget 2017;8:101383-93.

30. Makiyama A, Sagara K, Kawada J, et al. A randomized phase II study of weekly paclitaxel \pm trastuzumab in patients with HER2-positive advanced gastric or gastroesophageal junction cancer refractory to trastuzumab combined with fluoropyrimidine and platinum: WJOG7112G (T-ACT). J Clin Oncol 2018;36:4011.

31. Saeki H, Oki E, Kashiwada T, et al. Re-evaluation of HER2 status in patients with HER2-positive advanced or recurrent gastric cancer refractory to trastuzumab (KSCC1604). Eur J Cancer 2018;105:41-9.

32. Kim ST, Banks KC, Pectasides E, et al. Impact of genomic alterations on lapatinib treatment outcome and cell-free genomic landscape during HER2 therapy in HER2+ gastric cancer patients. Ann Oncol 2018;29:1037-48.

33. Klempner SJ, Chao J. Toward optimizing outcomes in Her2-positive gastric cancer: timing and genomic context matter. Ann Oncol 2018;29:801-2.

34. Janjigian YY, Sanchez-Vega F, Jonsson P, et al. Genetic predictors of response to systemic therapy in esophagogastric cancer. Cancer Discov 2018;8:49-58.

35. Wilke H, Muro K, Van Cutsem E, et al. Ramucirumab plus paclitaxel versus placebo plus paclitaxel in patients with previously treated advanced gastric or gastro-oesophageal junction adenocarcinoma (RAINBOW): a double-blind, randomised phase 3 trial. Lancet Oncol 2014;15:1224-35.

36. De Vita F, Borg C, Farina G, et al. Ramucirumab and paclitaxel in patients with gastric cancer and prior trastuzumab: subgroup analysis from RAINBOW study. Future Oncol 2019;15:2723-31.

37. Zhao D, Klempner SJ, Chao J. Progress and challenges in HER2-positive gastroesophageal adenocarcinoma. J Hematol Oncol 2019;12:50.

38. National LIbrary of Medicine. A Phase 2, Open-label,
Single-arm Trial of Trastuzumab Deruxtecan (DS 8201a) in HER2-positive, Unresectable or Metastatic Gastric or Gastro-esophageal Junction (GEJ) Adenocarcinoma Subjects Who Have Progressed on or After a Trastuzumab-containing Regimen Bethedsa, Maryland: ClinicalTrials.gov Identifier: NCT04014075; Available online: https://clinicaltrials.gov/ct2/show/study/NCT0401 4075 ? term $=$ NCT04014075\&draw $=2 \&$ rank $=1$

39. Fuchs CS, Doi T, Jang RW, et al. Safety and efficacy of pembrolizumab monotherapy in patients with previously treated advanced gastric and gastroesophageal junction cancer: Phase 2 clinical KEYNOTE-059 trial. JAMA Oncol 2018;4:e180013.

40. Kang YK, Boku N, Satoh T, et al. Nivolumab in patients with advanced gastric or gastro-oesophageal junction cancer refractory to, or intolerant of, at least two previous chemotherapy regimens (ONO-4538-12, ATTRACTION-2): a randomised, double-blind, placebocontrolled, phase 3 trial. Lancet 2017;390:2461-71.

41. Shitara K, Özgüroğlu M, Bang YJ, et al. Pembrolizumab versus paclitaxel for previously treated, advanced gastric or gastro-oesophageal junction cancer (KEYNOTE-061): a randomised, open-label, controlled, phase 3 trial. Lancet 2018;392:123-33.

42. Shitara K, Van Cutsem E, Bang YJ, et al. Pembrolizumab with or without chemotherapy vs. chemotherapy in patients with advanced G/GEJ cancer (GC) including outcomes according to Microsatellite Instability-High (MSI-H) status in KEYNOTE-062. Ann Oncol 2019;30:878-9.

43. Kojima T, Muro K, Francois E, et al. Pembrolizumab versus chemotherapy as second-line therapy for advanced esophageal cancer: Phase III KEYNOTE-181 study. J Clin Oncol 2019;37:2.

44. Kato K, Cho BC, Takahashi M, et al. Nivolumab versus chemotherapy in patients with advanced oesophageal squamous cell carcinoma refractory or intolerant to previous chemotherapy (ATTRACTION-3): a multicentre, randomised, open-label, phase 3 trial. Lancet Oncol 2019;20:1506-17.

45. Kim J, Bowlby R, Mungall AJ, et al. Integrated genomic characterization of oesophageal carcinoma. Nature 2017;541:169-75.

doi: $10.21037 / \operatorname{tgh} .2020 .01 .10$

Cite this article as: Epistola RJ, Chao J. Systemic therapy for advanced gastroesophageal cancers: progress and pitfalls. Transl Gastroenterol Hepatol 2020;5:53. 